\title{
Modelling Multi-Period Set-up Times in the Proportional Lot-Sizing Problem
}

\author{
Waldemar Kaczmarczyk ${ }^{\star}$
}

\begin{abstract}
This paper presents new mixed integer programming models for the Proportional Lot-Sizing Problem (PLSP) with set-up times longer than a period. Proposed models explicitly calculate the distribution of times amongst products in periods with a changeover and determine a final period for every set-up operation. Presented results prove that the proposed models are easier to solve using standard MIP methods than already known models.
\end{abstract}

Keywords: production, lot-sizing and scheduling, mixed integer programming

Mathematics Subject Classification: 90B30, 90C11

Revised: 11 December 2009

\section{INTRODUCTION}

This paper addresses mixed-integer programming (MIP) models of lot-sizing and scheduling problems, for several products with deterministic, dynamic demand, on a single machine with limited capacity. Models discussed are based on a model for the Proportional Lot-sizing and Scheduling Problem (PLSP) proposed by (Haase,1994), see also (Drexl and Haase, 1995). Here, new model formulations are presented which adapt the PLSP model to the case when set-up operations overlap multiple periods. The aim of this work is to develop models that allow practical examples to be solved with standard MIP methods.

Long set-up times are not the subject of many research papers, yet their importance may grow in the future. Increases in the computational capability of enterprise information systems allow more detailed planning, among other things planning based on a more detailed calendar. Thus, in the future, set-up times may become "longer" in relation to the time buckets used in planning (Suerie, 2006).

The assumption that a set-up operation cannot overlap multiple periods has two significant disadvantages. First, the time buckets have to be longer than the largest set-up time and this may prevent detailed planning. Long periods make it difficult to plan small lots, as they do not preserve a high utilization of period capacity.

\footnotetext{
* Department of Operations Research and Information Technology, AGH University of Science and Technology, Kraków, Poland. E-mail: waldek@agh.edu.pl
} 
Second, if the execution of set-up operations is limited by period boundaries, then some very good solutions may be cut off from the solution space. Suerie (2006) calculated the additional cost caused by the assumption that every set-up operation has to be executed within a single period for a set of random instances. For set-up times equal to $40 \%$ of period length, the total cost increment was $0.14 \%-0.49 \%$, depending on machine utilization. For two times longer set-up times, the cost increment was even $5 \%-8.37 \%$.

The PLSP model allows the processing of two products in a single period, one before and one after the set-up operation. Kimms and Drexl (1998) proposed a model formulation, which we denote PLSP/E, which uses variables that explicitly determine the distribution of time among products in periods with a changeover. It was originally used for a case with multiple resources. Models presented in this paper use such variables to determine in a direct way the amount of time used in every period to execute set-up operations. They assume that the period length is constant over the whole planning horizon, as is fully justified for short term, detailed planning.

The incentive to develop such models came from previous work on lot-sizing and scheduling models for identical parallel machines (Kaczmarczyk et al., 2006; Kaczmarczyk, 2010). On the one hand, there was a chance of finding models which are easier to solve, on the other hand, there is a need to extend models with parallel machines to the case with long set-up times. The development of models for a single machine is the first towards this goal.

The main objective of this paper is to find good model formulations that will make it possible to solve practical examples with standard MIP methods. To achieve that goal, it is necessary to define the problem using variables clearly describing crucial alternatives and to make the LP relaxation of the MIP problem as tight as possible (Williams, 2006, p. 197). An effective solution of a variety of lot-sizing problems depends crucially on the development of tight formulations for the special problem features occurring in practice (Belvaux and Wolsey, 2001; Pochet and Wolsey, 2006). It may therefore be favourable to extend the model by additional variables and constraints, to add important nodes to the search tree of the branch and bound algorithm and to make the LP relaxation as tight as possible.

There are only a few lot-sizing and scheduling models considering long set-up times. Cattrysse et al. (1993) and Blocher et al. (1999) adapted the Discrete Lot-sizing and Scheduling Problem (DLSP) to the case with times overlapping multiple periods. The DLSP restricts lot-sizes and set-up times to multiples of a period. Haase (1994) first proposed a PLSP model formulation which allowed set-up operations to overlap multiple periods, see also Drexl and Haase (1995). His model used variables that accumulate time assigned in consecutive periods to set-up operations. Suerie (2006) proved that this model is not correct and proposed two new models POST1 and POST2 to refine the idea of Haase. These two models used additional binary variables to define the start and end of set-up operations. Finally, Kaczmarczyk (2009) proposed a model based on the explicit formulation of PLSP which is the basis for the new formulations presented in this paper. General surveys on lot-sizing and scheduling models can be found in Drexl and Kimms (1997) or Jans and Degraeve (2008). 
The next section presents data parameters, variables and already known models. Section 3 presents new model formulations. Section 4 provides computational results. Finally, Section 5 gives a summary.

\section{KNOWN MODELS}

Parameters and variables common to all models discussed in this paper are presented in Tables 1 and 2. Parameters and variables specific to a single model are defined next to them.

Table 1. Parameters common to all models

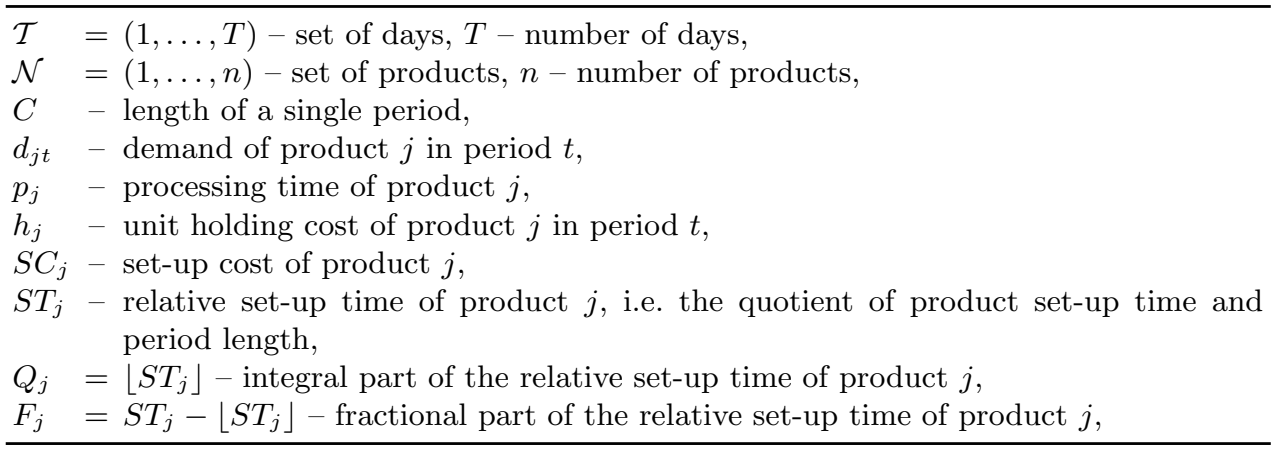

Table 2. Variables common to all models

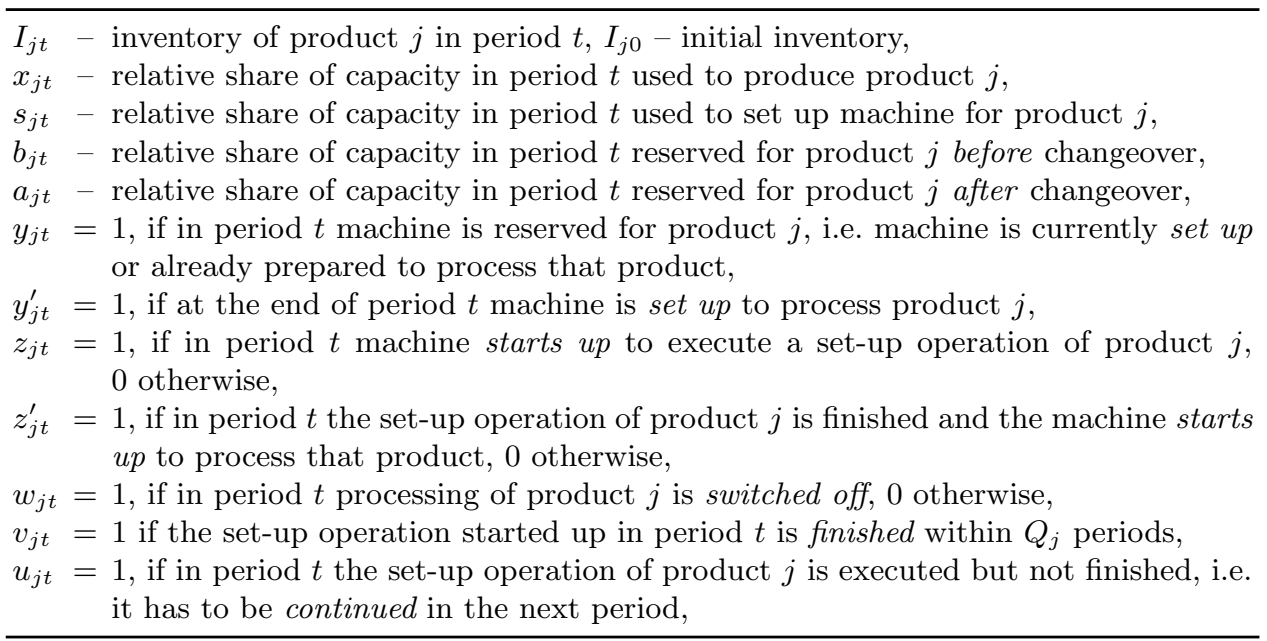

In all models described in this paper, all variables with period index $t \leqslant 0$ are assumed to be equal to 0 . All exceptions to this rule are stated explicitly. First, the inventory variables $I_{j 0}$ may represent non-zero initial inventories. Next, the set-up 
state variables $y_{j 0}$ may define the initial state of the machine. Another exception is described in the section about new models.

Table 3. Examples of a changeover in period $t$ from product $j$ to $k$

\begin{tabular}{|c|c|c|c|c|}
\hline set-up & $s$ & $t-1$ & $t$ & $t+1$ \\
\hline state & $y_{j s}^{\prime}$ & 1 & & \\
\hline \multirow{3}{*}{$\begin{array}{l}\text { switch-off } \\
\text { start-up }\end{array}$} & $w_{j s}$ & & 1 & \\
\hline & $z_{k s}$ & & 1 & \\
\hline & $z_{k s}^{\prime}$ & & 1 & \\
\hline \multirow{4}{*}{$\begin{array}{l}\text { execution } \\
\text { continuation } \\
\text { state }\end{array}$} & $s_{k s}$ & & 0.5 & \\
\hline & $u_{k s}$ & & & \\
\hline & $y_{k s}$ & & 1 & 1 \\
\hline & $y_{k s}^{\prime}$ & & 1 & 1 \\
\hline
\end{tabular}

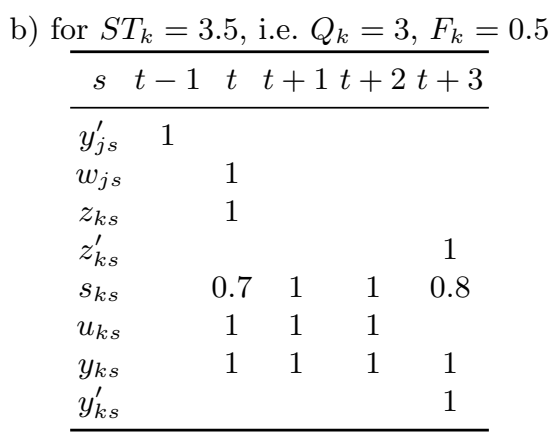

In Table 3 two examples are presented, illustrating definitions of some variables. There are two kinds of set-up state variables, $y_{j t}$ and $y_{j t}^{\prime}$, and two kinds of startup variables, $z_{j t}$ and $z_{j t}^{\prime}$. These variables are used in the presented models in three different combinations. For many constraints, differences between them are unimportant and such constraints are described as equivalent, independently of the kind of variables used in them. The form of some other types of constraints depends on the choice of these variables and such constraints are described in detail.

For products with set-up times shorter than the period length, i.e. $Q_{j}=0$, if the whole set-up operation is executed within a single period, there is no difference between the two types of set-up and start-up variables. In all other cases the pair of variables $u_{j t}$ and $y_{j t}^{\prime}$ constitute an alternative to variable $y_{j t}$.

\subsection{STANDARD PLSP MODEL}

Equations (1) present the classic PLSP model (Drexl and Haase, 1995). This model is based on the assumption that set-up times are smaller than period capacity. It does not directly calculate processing times before and after a set-up operation.

$$
\begin{array}{cl}
\min \sum_{t \in \mathcal{T}} \sum_{j \in \mathcal{N}}\left(S C_{j} z_{j t}+h_{j t} I_{j t}\right) & \\
I_{j t-1}+\frac{C}{p_{j}} x_{j t}-d_{j t}=I_{j t}, & t \in \mathcal{T}, j \in \mathcal{N} \\
B_{j t}\left(y_{j, t-1}+y_{j t}\right) \geqslant x_{j t}, & t \in \mathcal{T}, j \in \mathcal{N} \\
\sum_{j \in \mathcal{N}}\left(x_{j t}+S T_{j} z_{j t}\right) \leqslant 1, & t \in \mathcal{T} \\
\sum_{j \in \mathcal{N}} y_{j t}=1, & t \in \mathcal{T} \\
y_{j t}-y_{j, t-1} \leqslant z_{j t}, & t \in \mathcal{T}, j \in \mathcal{N}
\end{array}
$$




$$
\begin{aligned}
I_{j t} & \geqslant 0, & & t \in \mathcal{T}, j \in \mathcal{N} \\
x_{j t}, z_{j t} & \in[0,1], & & t \in \mathcal{T}, j \in \mathcal{N} \\
y_{j t} & \in\{0,1\}, & & t \in \mathcal{T}, j \in \mathcal{N}
\end{aligned}
$$

where $B_{j t}=\min \left\{1, p_{j} / C \sum_{s=t}^{T} d_{j s}\right\}$ is the minimum of period capacity and rest demand.

Constraint (1.2) describes the balance of inventory, production and demand. Constraint (1.3) allows production to take values higher than zero only if a machine is set up to process a given product in the current or previous period. Inequality (1.4) ensures that total production and set-up operations do not exceed period capacity. Constraint (1.5) ensures that in every period, the set up state variable of only one product takes the value one, i.e. the machine is set up to process exactly one product. Equality (1.6) ensures that start-up variables take the value one if machine is set up to process a given product.

The start-up variables do not have to be binary, because their values are minimized and constraint (1.6) defines their lower bound which is equal either 1 or 0 . Nevertheless they are usually defined as binary. First, because they represent important branching nodes of the branch and bound algorithm. Next, because in model formulations for special problem features, they are often involved in more complex relations with other variables which could lead to fractional values.

\subsection{VALID INEQUALITIES}

Valid inequalities are constraints which are not necessary to define a correct model but make it more tight (Belvaux and Wolsey, 2001; Wolsey, 2002; Pochet and Wolsey, 2006, p. 97). First, they ensure that in linear relaxations of the model, integer variables are more likely to take integer values. Next, they improve the lower bounds of nodes in the branch and bound algorithm. In this way, valid inequalities decrease the number of nodes needed to solve a MIP problem and may speed up execution of the branch and bound algorithm.

On the one hand, valid inequalities tighten the model, but on the other hand, they also increase its size and each iteration of the algorithm takes more time. Thus, it is very important to find a good balance between model tightness and size.

Commercial branch-and-cut systems are able to generate some of the valid inequalities automatically as cutting planes. Thus, it is very hard to predict which of them will be useful and which won't.

Valid inequalities applied to the models being discussed are presented below (Belvaux and Wolsey, 2001; Wolsey, 2002; Pochet and Wolsey, 2006):

$$
\begin{array}{cl}
z_{j t} \leqslant y_{j t}, & t \in \mathcal{T}, j \in \mathcal{N} \\
z_{j t} \leqslant 1-y_{j, t-1}, & t \in \mathcal{T}, j \in \mathcal{N} \\
I_{j, t-1} \geqslant \sum_{s=t}^{t+p} d_{j s}\left[1-y_{j, t-1}-\sum_{r=t}^{s} z_{j r}\right], & t \in \mathcal{T}, p=0, \ldots, T-t, j \in \mathcal{N}
\end{array}
$$


Constraint (1.10) allows a start-up variable to take the value one only if the machine is set up to process a given product, and constraint (1.11) only allows it if in the previous period the machine was processing some other product. Constraint (1.12) determines the inventory level at the end of period $t-1$ necessary to satisfy total demand, from period $t$ up to period $t+p$, if no production takes place in that interval. Similar valid inequalities play a major role in tightening many lot-sizing and scheduling models. To limit the number of such constraints, in all the following models parameter $p$ does not exceed the value 5 .

\subsection{PLSP-POST1 MODEL}

The first model proposed by Suerie (2006), denoted PLSP-POST1, uses one additional continuous variable $s_{j t}^{c}$ which describes the set-up time in multiple periods belonging to the same set-up operation accumulated up to period $t$. This is the first model which uses the binary set-up continuation variables $u_{j t}$. The POST1 model is stated as follows:

$$
\begin{aligned}
& \min \sum_{t \in \mathcal{T}} \sum_{j \in \mathcal{N}}\left(S C_{j} z_{j t}^{\prime}+h_{j t} I_{j t}\right) \\
& I_{j t-1}+\frac{C}{p_{j}} x_{j t}-d_{j t}=I_{j t}, \quad t \in \mathcal{T}, j \in \mathcal{N} \\
& \sum_{j \in \mathcal{N}}\left(x_{j t}+s_{j t}\right) \leqslant 1, \quad t \in \mathcal{T} \\
& \sum_{j \in \mathcal{N}}\left(y_{j t}^{\prime}+u_{j t}\right)=1, \quad t \in \mathcal{T} \\
& y_{j t}^{\prime}-y_{j, t-1}^{\prime} \leqslant z_{j t}^{\prime}, \quad t \in \mathcal{T}, j \in \mathcal{N} \\
& B_{j t}\left(y_{j, t-1}^{\prime}+z_{j t}^{\prime}\right) \geqslant x_{j t}, \quad t \in \mathcal{T}, j \in \mathcal{N} \\
& s_{j, t-1}^{c}+s_{j t} \geqslant s_{j t}^{c}, \quad t \in \mathcal{T}, j \in \mathcal{N} \\
& S T_{j} z_{j t}^{\prime} \leqslant s_{j t}^{c}, \quad t \in \mathcal{T}, j \in \mathcal{N} \\
& s_{j t}+S T_{j}\left(1-\sum_{k \in \mathcal{N} \backslash\{j\}}\left(y_{k, t-1}^{\prime}+u_{k, t-1}\right)\right) \geqslant s_{j t}^{c}, \quad t \in \mathcal{T}, j \in \mathcal{N} \\
& s_{j t}+S T_{j}\left(1-\sum_{k \in \mathcal{N} \backslash\{j\}} z_{k t}^{\prime}\right) \geqslant s_{j t}^{c}, \quad t \in \mathcal{T}, j \in \mathcal{N} \\
& 1-\sum_{k \in \mathcal{N} \backslash\{j\}} z_{k t}^{\prime} \geqslant y_{j t}^{\prime}, \quad t \in \mathcal{T}, j \in \mathcal{N} \\
& z_{j t}^{\prime}+u_{j t} \geqslant s_{j t}, \quad t \in \mathcal{T}, j \in \mathcal{N} \\
& I_{j t}, s_{j t}^{c} \geqslant 0, \quad t \in \mathcal{T}, j \in \mathcal{N}
\end{aligned}
$$




$$
\begin{aligned}
x_{j t}, s_{j t} & \in[0,1], & & t \in \mathcal{T}, j \in \mathcal{N} \\
y_{j t}^{\prime}, z_{j t}^{\prime}, u_{j t} & \in\{0,1\}, & & t \in \mathcal{T}, j \in \mathcal{N}
\end{aligned}
$$

Let's describe all the differences between the above model and the standard PLSP model (1). Constraint (2.4) replaces constraint (1.5) to take into account the set-up continuation variables. Set-up constraints (2.6) are similar to set-up constraints (1.3), but production is possible now if either a set-up operation has been finished in period $t$, i.e. $z_{j t}^{\prime}=1$, or the machine was set up to process product $j$ in period $t-1$, i.e. $y_{j, t-1}^{\prime}=1$. Constraints $(2.7)-(2.12)$ accumulate set-up execution times.

To make the model formulation tighter, two valid inequalities (2.16) and (2.17) are added:

$$
\begin{array}{r}
1-\sum_{k \in \mathcal{N} \backslash\{j\}} u_{k, t-1} \geqslant z_{j t}^{\prime}, \quad t \in \mathcal{T}, j \in \mathcal{N} \\
1-\sum_{k \in \mathcal{N} \backslash\{j\}} y_{k t}^{\prime} \geqslant z_{j t}^{\prime}, \quad t \in \mathcal{T}, j \in \mathcal{N}
\end{array}
$$

\subsection{PLSP-POST2 MODEL}

The second model proposed by (Suerie, 2006), denoted PLSP-POST2, uses two types of set-up execution variables $s_{j t}^{1}$ and $s_{j t}^{2}$, which hold the relative share of set-up time. Variable $s_{j t}^{2}$ holds the set-up time in all periods in which a set-up operation is executed except the last one, i.e. it is used if the set-up operation has to be continued in the next period. Variable $s_{j t}^{1}$ holds the set-up time only in the period in which the set-up operation is finished. Variables $s_{j t}^{1}$ and $s_{j t}^{2}$ have to be discriminated from variable $s_{j t}$ used in all models presented in later sections, which gives the relative share of a period capacity used for set-up execution. Parameter $s p_{j}$ represents the maximum number of periods involved in the execution of a single set-up operation, i.e. for constant capacity $s p_{j}=Q_{j}+1$.

With these definitions, the POST2 model can be stated as follows:

$$
\begin{aligned}
\min \sum_{t \in \mathcal{T}} \sum_{j \in \mathcal{N}}\left(S C_{j} z_{j t}^{\prime}+h_{j t} I_{j t}\right) & \\
I_{j t-1}+\frac{C}{p_{j}} x_{j t}-d_{j t}=I_{j t}, & t \in \mathcal{T}, j \in \mathcal{N} \\
\sum_{j \in \mathcal{N}}\left(x_{j t}+S T_{j}\left(s_{j t}^{1}+s_{j t}^{2}\right)\right) \leqslant 1, & t \in \mathcal{T} \\
\sum_{j \in \mathcal{N}} y_{j t}^{\prime} \leqslant 1, & t \in \mathcal{T} \\
y_{j t}^{\prime}-y_{j, t-1}^{\prime} \leqslant z_{j t}^{\prime}, & t \in \mathcal{T}, j \in \mathcal{N} \\
B_{j t}\left(y_{j, t-1}^{\prime}+z_{j t}^{\prime}\right) \geqslant x_{j t}, & t \in \mathcal{T}, j \in \mathcal{N}
\end{aligned}
$$




$$
\begin{aligned}
s_{j t}^{1}+\sum_{s=t+1-s p_{j}}^{t-1} s_{j s}^{2} \geqslant y_{j t}^{\prime}, & t \in \mathcal{T}, j \in \mathcal{N} \\
\sum_{j \in \mathcal{N}}\left(y_{j t}^{\prime}+s_{j t}^{2}\right) \leqslant 1, & t \in \mathcal{T} \\
1-\sum_{k \in \mathcal{N} \backslash\{j\}} s_{k, t-1}^{2} \geqslant y_{j t}^{\prime}, & t \in \mathcal{T}, j \in \mathcal{N} \\
1-\sum_{k \in \mathcal{N} \backslash\{j\}} z_{k t}^{\prime} \geqslant y_{j t}^{\prime}, & t \in \mathcal{T}, j \in \mathcal{N} \\
\sum_{k \in \mathcal{N} \backslash\{j\}} z_{k t}^{\prime}+\sum_{s=0} z_{j, t+s}^{\prime} \leqslant 1, & t \in \mathcal{T}, j \in \mathcal{N}: s p_{j} \geqslant 4 \\
I_{j t}, \geqslant 0, & t \in \mathcal{T}, j \in \mathcal{N} \\
x_{j t}, s_{j t}^{1}, s_{j t}^{2} \in[0,1], & t \in \mathcal{T}, j \in \mathcal{N} \\
z_{j t}^{\prime}, y_{j t}^{\prime} \in\{0,1\}, & t \in \mathcal{T}, j \in \mathcal{N}
\end{aligned}
$$

The basis of this formulation constitutes the standard PLSP model. Let's describe all the differences. Capacity constraints (3.3) use new set-up execution variables. Constraints (3.4) replace (1.5) to take into consideration periods when none of the products can be produced. Constraints (3.6) and (3.10) are the same as constraints (2.6) and (2.11) of the POST1 model. Constraints (3.7)-(3.11) preserve appropriate values of set-up execution variables.

The above formulation of the PLSP-POST2 model differs from the original (Suerie, 2006). Here, the unique set-up constraint (3.4) is an inequality because in some periods none of the set-up state variables $y_{j t}^{\prime}$ is equal to 1 . In the original model, idle periods are assigned to a fictitious product without demand.

The POST2 model is less restrictive than the POST1 model because it does not use the set-up continuation variable. For this reason, POST2 may preserve better values of the objective function than POST1.

Table 4. Example of solution for $S T_{j}=S T_{k}=0.5$ which is infeasible in POST1

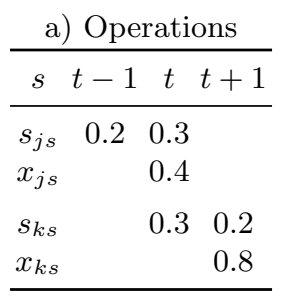

\begin{tabular}{lccc}
\multicolumn{3}{c}{ b) } & POST1 \\
\hline$s$ & $t-1$ & $t$ & $t+1$ \\
\hline$u_{j s}$ & 1 & & \\
$y_{j s}^{\prime}$ & & 1 & \\
$u_{k s}$ & & 1 & \\
$y_{k s}^{\prime}$ & & & 1 \\
\hline
\end{tabular}

An example illustrating the difference is presented in Table 4 . In period $t$, the set-up operation of product $j$ is finished, some volume of that product is processed, and before the end of that period the set-up operation of product $k$ starts. Such a solution is not feasible in the POST1 model because constraint 2.4 does not allow it: 
$y_{j t}^{\prime}+u_{k t}=2$. Such small lots may be useful if there are small demand values isolated (in time) from the rest of the demand of the same product.

\subsection{CSLP MODEL FOR LONG SET-UP TIMES}

The model known as the Continuous Set-up Lot-sizing Problem (CSLP) assumes that in every period only one product may be produced, which is equivalent to the assumption that every set-up operation starts at the beginning of a period. CSLP is more restrictive than PLSP but much easier to solve. The results of CSLP are used in this paper to evaluate the results of the PLSP model. To build the CSLP from the PLSP model (1) one has to substitute constraints (1.3) and (1.4) with the following one:

$$
x_{j t}+S T_{j} z_{j t} \leqslant y_{j t}, \quad t \in \mathcal{T}, j \in \mathcal{N}
$$

The adaptation of the CSLP model to the case with set-up times longer than the period using variables $y_{j t}$, which we call CSLP-LST, is a straightforward task. First, in the capacity constraint 4.1 the expression $S T_{j} z_{j t}$ is replaced with the variable $s_{j t}$ :

$$
x_{j t}+s_{j t} \leqslant y_{j t}, \quad t \in \mathcal{T}, j \in \mathcal{N}
$$

Next it is necessary to add the following constraints to determine the values of $s_{j t}$ :

$$
\begin{aligned}
\sum_{r=1}^{Q} z_{j, t-(r-1)} & \leqslant s_{j t}, & & t \in \mathcal{T}, j \in \mathcal{N} \\
F_{j} * z_{j, t-Q_{j}} & \leqslant s_{j t}, & & t \in \mathcal{T}, j \in \mathcal{N} \\
s_{j t} & \in[0,1], & & t \in \mathcal{T}, j \in \mathcal{N}
\end{aligned}
$$

Constraint (4.3) reserves full capacity for a set-up operation in the first $Q_{j}$ periods and constraint (4.4) calculates $s_{j t}$ during the period in which the set-up operation is finished.

Valid inequalities (1.10) and (1.11) can be applied in this model without modifications. The inventory lower bound constraints (1.12) have to be modified because long set-up times prevent production in many periods:

$$
\begin{aligned}
I_{j, t-1} \geqslant \sum_{s=t}^{t+p} d_{j s}\left[1-\left(y_{j, t-1}-\sum_{r=1}^{Q_{j}} z_{j, t-(r-1)}\right)-\sum_{r=t+1}^{s} z_{j, r-Q_{j}}\right] & \\
& t \in \mathcal{T}, p=0, \ldots, T-t, j \in \mathcal{N}
\end{aligned}
$$

Constraint (4.6) differs from (1.12) for two reasons. First, the CSLP-LST model uses the set-up state variables $y_{j, t-1}$ which take the value 1 also in periods in which production is not allowed, i.e. when the whole period might be used to execute the set-up operation. Variables $z_{j, t-(r-1)}$ (in round parenthesis) cancel $y_{j, t-1}$ if production is not possible in $t-1$. Second, the production is now possible after $Q_{j}$ periods counted from the start-up period. Thus, the start-up variables at the end of the constraint are shifted back by $Q_{j}$ periods. 


\subsection{EXPLICIT PLSP MODEL}

Kimms and Drexl (1998) proposed a PLSP model with multiple resources, i.e. for products which require several resources at the same time. It uses variables directly describing production volume before and after a set-up operation. A model is presented below for a single resource (5) based on the same idea. We call it the explicit PLSP model, i.e. PLSP/E:

$$
\begin{array}{rlrl}
\min & \sum_{t \in \mathcal{T}} \sum_{j \in \mathcal{N}}\left(S C_{j} z_{j t}+h_{j t} I_{j t}\right) & \\
I_{j, t-1}+\frac{C}{p_{j}} x_{j t}-d_{j t} & =I_{j t}, & & \\
x_{j t}+S T_{j} z_{j t} & \leqslant y_{j t}-z_{j t}+b_{j t}+a_{j t}, & & t \in \mathcal{T}, j \in \mathcal{N}, j \in \mathcal{N} \\
\sum_{j \in \mathcal{N}} y_{j t} & =1, & & t \in \mathcal{T} \\
z_{j t}-w_{j t} & =y_{j t}-y_{j, t-1}, & & t \in \mathcal{T}, j \in \mathcal{N} \\
\sum_{j \in \mathcal{N}}\left(b_{j t}+a_{j t}\right) & =\sum_{j \in \mathcal{N}} z_{j t}, & & t \in \mathcal{T} \\
a_{j t} \leqslant z_{j t}, & t \in \mathcal{T}, j \in \mathcal{N} \\
b_{j t} \leqslant w_{j t}, & t \in \mathcal{T}, j \in \mathcal{N} \\
I_{j t} \geqslant 0, & t \in \mathcal{T}, j \in \mathcal{N} \\
y_{j t}, z_{j t} \in\{0,1\}, & t \in \mathcal{T}, j \in \mathcal{N} \\
x_{j t}, a_{j t}, b_{j t}, w_{j t} \in[0,1], & t \in \mathcal{T}, j \in \mathcal{N}
\end{array}
$$

Constraint (5.3) limits the production volume. The set-up state variables $y_{j t}$ on the right-hand side of the constraint determines the capacity assigned to product $j$. This is untrue only in periods with a changeover. If the machine in the current period is started up to process product $j$, the capacity is described by variable $a_{j t}$ and the value of the set-up state variables $y_{j t}$ has to be cancelled by the start-up variable $z_{j t}$. If during the current period, the machine is switched off from processing product $j$, the capacity is described by variable $b$, while all other variables on the right-hand side are equal to zero.

Constraints (5.6) ensure that the sum of variables $b_{j t}$ and $a_{j t}$ is equal to the period, no more and no less. Constraints (5.7) and (5.8) allow non-zero values of $b_{j t}$ and $a_{j t}$ only if the machine is respectively switched off or started up to process product $j$.

The original formulation of this model (Kimms and Drexl, 1998) is slightly different than the PLSP/E model presented above (5). First, PLSP/E takes into account only a single resource and considers set-up operations (times) processed within a single period. Next, the right-hand side of constraint (5.8) includes the switch-off variable $w_{j t}$ while in the original model it includes the set-up state variable $y_{j, t-1}$. Finally the original variables $b_{j t}$ and $a_{j t}$ describe production volumes and not relative shares of capacity. 


\subsection{EXPLICIT PLSP MODEL WITH SET-UP FINISH VARIABLES}

The first model with long set-up times based on PLSP/E, which we denote PLSP/EMS/F, was proposed by Kaczmarczyk (2009). It determines directly the time reserved in all periods for set-up operations using $b_{j t}$ and $a_{j t}$ variables. The whole model is presented below, including the valid inequalities that are applied.

PLSP/E-MS/F contains all the constraints of the PLSP/E model with only one modification. In the the capacity constraint (5.3), expression $S T_{j t} z_{j t}$ is replaced by variable $s_{j t}$ :

$$
\begin{array}{rlrl}
x_{j t}+s_{j t} & \leqslant y_{j t}-z_{j t}+b_{j t}+a_{j t}, & & t \in \mathcal{T}, j \in \mathcal{N} \\
s_{j t} & \in[0,1], & t \in \mathcal{T}, j \in \mathcal{N}
\end{array}
$$

Table 5. Two cases of set-up execution for $S T_{j}=3.5$, i.e. $Q_{j}=3$ and $F_{j}=0.5$

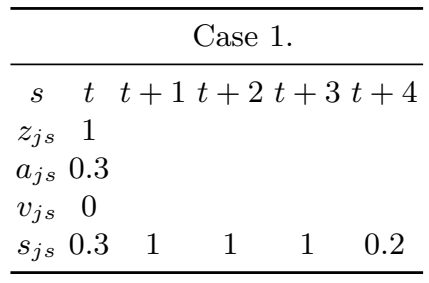

\begin{tabular}{ccccc}
\hline & \multicolumn{3}{c}{ Case 2.} \\
\hline$s$ & $t$ & $t+1$ & $t+2$ & $t+3 t+4$ \\
$z_{j s}$ & 1 & & & \\
$a_{j s}$ & 0.7 & & & \\
$v_{j s}$ & 1 & & & \\
$s_{j s}$ & 0.7 & 1 & 1 & 0.8 \\
\hline
\end{tabular}

In Table 5, two cases are presented for the set-up execution of products with setup time longer than the period, i.e. $Q_{j} \geqslant 1$. If the amount of time after changeover $a_{j t}$ is shorter than the remainder of the relative set-up time $F_{j}$, see case a), then the set-up operation is spread over $Q+1$ periods, otherwise over $Q$ periods, see case b). This is the basic idea of the model. It is correct only for a constant period capacity $C$ over the whole planning horizon.

The following constraint ensures that the set-up finish variable $v_{j t}$ takes the value 1 if the set-up operation overlaps $Q$ periods:

$$
\begin{aligned}
a_{j t}-F_{j} & \leqslant v_{j t}, & & t \in \mathcal{T}, j \in \mathcal{N} \\
v_{j t} & \in\{0,1\}, & t & \in \mathcal{T}, j \in \mathcal{N}
\end{aligned}
$$

The constraints below retain the correct values in the set-up execution variables $s_{j t}$ if the set-up times are shorter than the period, i.e. with $Q_{j}=0$ :

$$
\begin{aligned}
F_{j} v_{j t} & \leqslant s_{j t}, & & t \in \mathcal{T}, j \in \mathcal{N}: Q_{j}=0 \\
a_{j t}-v_{j t} & \leqslant s_{j t}, & & t \in \mathcal{T}, j \in \mathcal{N}: Q_{j}=0 \\
F_{j} z_{j, t-1}-a_{j, t-1} & \leqslant s_{j t}, & t & \in \mathcal{T}, j \in \mathcal{N}: Q_{j}=0
\end{aligned}
$$

If $Q_{j}=0$ then a set-up operation may overlap at most two periods. Constraints (6.5) and (6.6) keep the correct values of $s_{j t}$ during periods in which changeovers occur. First, if the whole set-up operation is executed within a single period, and second, 
if it overlaps two periods. Constraint (6.7) determines the amount of time needed to complete the set-up operations in the second period.

The constraints below retain the correct values of the set-up execution variables $s_{j t}$, if the set-up times are longer than the period, i.e. with $Q_{j} \geqslant 1$ :

$$
\begin{aligned}
a_{j t} \leqslant s_{j t}, & & t \in \mathcal{T}, j \in \mathcal{N}: Q_{j} \geqslant 1 \\
\sum_{r=1}^{Q_{j}-1} z_{j, t-r} \leqslant s_{j t}, & & t \in \mathcal{T}, j \in \mathcal{N}: Q_{j} \geqslant 1 \\
\left(F_{j}+1\right) v_{j, t-Q_{j}}-a_{j, t-Q_{j}} \leqslant s_{j t}, & & t \in \mathcal{T}, j \in \mathcal{N}: Q_{j} \geqslant 1 \\
z_{j, t-Q_{j}}-v_{j, t-Q_{j}} \leqslant s_{j t}, & & t \in \mathcal{T}, j \in \mathcal{N}: Q_{j} \geqslant 1 \\
F_{j} z_{j, t-\left(Q_{j}+1\right)}-a_{j, t-\left(Q_{j}+1\right)} \leqslant s_{j t}, & & t \in \mathcal{T}, j \in \mathcal{N}: Q_{j} \geqslant 1
\end{aligned}
$$

Consecutive periods which may be involved in a set-up operation may be divided into four different kinds. Let us discuss the examples in Table 5 assuming that the changeover occurs in period $t$. In that period, set-up execution always lasts exactly $a_{j t}$ and this preserves constraint (6.8). Constraint (6.9) ensures that the next $Q_{j}-1$ periods are always fully used by the set-up operation, i.e. $s_{j t}=1$. In the example, these are periods $t+1$ and $t+2$. The use of the two last periods depends on value of the set-up finish variable $v_{j t}$. Constraint (6.10) determines the set-up execution time in period $t+Q_{j}$ if $v_{j t}=1$, constraint (6.11) in the other case. If $v_{j t}=0$, constraint (6.12) calculates the value of the set-up execution variable in period $t+\left(Q_{j}+1\right)$. In the other case $s_{j, t+\left(Q_{j}+1\right)}=0$.

Besides the valid inequalities (1.10) and (1.11), the following constraint is also useful:

$$
v_{j t} \leqslant z_{j t}, \quad t \in \mathcal{T}, j \in \mathcal{N}
$$

Inequality (6.13) ensures that variable $v_{j t}$ takes the value 1 only in periods with a start-up. This is important for the accuracy of the next valid inequality.

Inventory lower bound constraints (4.6) from the CSLP-LST model can be applied here with some modifications:

$$
\begin{aligned}
I_{j, t-1} \geqslant \sum_{s=t}^{t+p} d_{j s}[1 & -\left(y_{j, t-1}-\sum_{r=1}^{Q_{j}} z_{j,(t-1)-(r-1)}-v_{j,(t-1)-Q_{j}}\right) \\
& \left.-\left(\sum_{r=t}^{s} z_{j, r-\left(Q_{j}+1\right)}+v_{j, s-Q_{j}}\right)\right], \quad t \in \mathcal{T}, p=0, \ldots, T-t, j \in \mathcal{N}
\end{aligned}
$$

Constraint (6.14) differs from (4.6) for one reason. In the PLSP model, production in period $t$ is possible if the set-up operation started up at least $Q_{j}+1$ periods earlier or in period $t-Q_{j}$. But in the last case, production is allowed only if the set-up time in the start-up period is long enough, i.e. $v_{j t}=1$ (compare constraint (6.3)). In this way, indices in the start-up variables $z_{j t}$ have been decreased by 1 and the set-up finish variables $v_{j t}$ have been introduced. 
The PLSP/E-MS/F model preserves the same values as the POST2 model with one small exception. Table 6 presents an example of two start-ups in the same period where the first set-up operation starts at the beginning of the period. This is a feasible solution in the POST2 model (as it is also in the POST1 model). In the E-MS/F model, a regular solution is infeasible, see Table $6 \mathrm{c}$ ), because at most one set-up state variable can be equal to 1 at the same time.

Table 6. Example of two start-ups in the same period for products with $S T_{j}=S T_{k}=0.2$

\begin{tabular}{|c|c|c|c|c|c|c|}
\hline a) $\mathrm{Op}$ & perations & b) POST2 & c) infeasible E-MS/F & d) fec & asible E-I & $\mathrm{MS} / \mathrm{F}$ \\
\hline$s$ & $t \quad t+1$ & $s \quad t t+1$ & $s \quad t t+1$ & $s$ & $t-1 t t$ & +1 \\
\hline$s_{j s} 0$ & & $z_{j s}^{\prime} 1$ & $z_{j s} 1$ & $z_{j s}$ & 1 & \\
\hline$x_{j s} 0$ & 0.7 & $y_{j s}^{\prime} 1$ & $y_{j s} 1$ & $y_{j s}$ & 1 & \\
\hline$s_{k s} 0$ & 0.10 .1 & $z_{k s}^{\prime}$ & $z_{k s} 1$ & $z_{k s}$ & 1 & \\
\hline$x_{k s}$ & 0.9 & $y_{k s}^{\prime}$ & $y_{k s} 1$ & $y_{k s}$ & 1 & 1 \\
\hline
\end{tabular}

Such a solution is possible in the E-MS/F model only thanks to a small trick, presented in Table $6 \mathrm{~d}$ ). One has to start up operation in period $t-1$, but with execution time $s_{j, t-1}=0$, and the whole set-up is executed in period $t$. This trick however does not work in the first period as long as that start-up and state variables are set to zero in period zero. To make it work, it is necessary to allow positive values for both variables but to keep set-up execution variables equal to zero, i.e. $s_{j 0}=0$.

Suerie (2006) noticed that in the POST1 model it is possible to process during period $t$ yet another product, before product $j$.

\section{NEW MODELS}

The new models use the set-up continuation variables $u_{j t}$ introduced by Suerie (2006) in the PLSP-POST1 model to discriminate periods fully used to execute set-up operations from periods where production is possible.

\subsection{EXPLICIT PLSP MODEL WITH SET-UP CONTINUATION VARIABLES}

In the first new model, denoted PLSP/E-MS/C, the set-up finish $v_{j t}$ variable is replaced by the set-up continuation variables $u_{j t}$. Therefore, one can use here the set-up state variable $y_{j t}^{\prime}$ which is equal to 1 only in periods in which production is possible. All differences between the E-MS/F and E-MS/C models result from this single change in the set of variables. All constraints of the new model are mentioned below, but only those which differ from the constraints of the E-MS/F model will be described in detail.

In the absence of the set-up finish variable, constraint (6.3) takes the following form:

$$
\begin{aligned}
F_{j} z_{j t}-a_{j t} & \geqslant u_{j, t+Q_{j},} & & j \in \mathcal{N}, t \in \mathcal{T}: t \leqslant T-Q_{j} \\
u_{j t} & \in\{0,1\}, & & j \in \mathcal{N}, t \in \mathcal{T}
\end{aligned}
$$


If there is a start-up in period $t$, i.e. $z_{j t}=1$, the set-up continuation variables play a dual role. First, variable $u_{j, t+Q_{j}}$ describes a decision if the set-up operation overlaps period $t+\left(Q_{j}+1\right)$. Second, variables $u_{j, t+1} \ldots u_{j, t+Q_{j}-1}$, and eventually $u_{j, t+Q_{j}}$, contain information on whether the machine is busy with a set-up operation and production is not possible.

Below constraints are presented for products with short set-up times, i.e. $Q_{j}=0$, which differ from constraints in the E-MS/F model:

$$
x_{j t}+s_{j t} \leqslant y_{j t}^{\prime}+u_{j t}-z_{j t}+b_{j t}+a_{j t}, \quad t \in \mathcal{T}, j \in \mathcal{N}: Q_{j}=0
$$

In capacity constraint (7.3), compared to (6.1), the set-up continuation variable $u_{j t}$ is introduced. It cancels the start-up variable $z_{j t}$ in the case when set-up operation overlaps two periods and $y_{j t}^{\prime}=0$.

To replace constraints $(6.5)-(6.7)$ it is necessary to substitute the set-up finish variable $v_{j t}$ with expressions involving the set-up continuation variable $u_{j t}$ and the start-up variable $z_{j t}$. The resulting constraints are presented below:

$$
\begin{array}{rlrl}
F_{j}\left(z_{j t}-u_{j t}\right) & \leqslant s_{j t}, & t \in \mathcal{T}, j \in \mathcal{N}: Q_{j}=0 \\
a_{j t}-\left(1-u_{j t}\right) \leqslant s_{j t}, & t \in \mathcal{T}, j \in \mathcal{N}: Q_{j}=0 \\
F_{j} z_{j, t-1}-a_{j, t-1} \leqslant s_{j t}, & t \in \mathcal{T}, j \in \mathcal{N}: Q_{j}=0
\end{array}
$$

The following capacity constraint for products with set-up times shorter than the period, i.e. with $Q_{j} \geqslant 1$, is shorter than (6.1), because processing during periods fully utilized by the set-up operation is now prevented by the set-up continuation variables:

$$
x_{j t}+s_{j t} \leqslant y_{j t}^{\prime}+b_{j t}, \quad t \in \mathcal{T}, j \in \mathcal{N}: Q_{j} \geqslant 1
$$

Constraints from the E-MS/F model which determine the set-up execution variable $s_{j t}$ for products with $Q_{j} \geqslant 1$, i.e. (6.8)-(6.12), are substituted by the following constraints, which use the set-up continuation variables $u_{j t}$ in place of the set-up finish variables $v_{j t}$ :

$$
\begin{aligned}
\sum_{r=1}^{Q_{j}} z_{j, t-(r-1)} \leqslant u_{j t}, & t \in \mathcal{T}, j \in \mathcal{N}: Q_{j} \geqslant 1 \\
\left(F_{j}+1\right) z_{j, t-Q_{j}}-a_{j, t-Q_{j}} \leqslant s_{j t}, & t \in \mathcal{T}, j \in \mathcal{N}: Q_{j} \geqslant 1 \\
F_{j} z_{j, t-\left(Q_{j}+1\right)}-a_{j, t-\left(Q_{j}+1\right)} \leqslant s_{j t}, & t \in \mathcal{T}, j \in \mathcal{N}: Q_{j} \geqslant 1
\end{aligned}
$$

An equivalent of constraint (6.8) is superfluous, because production in period when the set-up operation starts is now prevented by $u_{j t}=1$ and $y_{j t}=0$. Constraint (7.8), which replaces $(6.9)$, now fixes the set-up continuation variables $u_{j t}$. It ensures that $u_{j t}$ are always equal to 1 in the first $Q_{j}-1$ periods after the period with changeover.

Constraint (6.11) is here superfluous because in this model the set-up continuation variable determined by constraint (7.1) fully prevents production in such a situation. Constraint (7.9) differs from $(6.10)$ because $v_{j, t-Q_{j}}$ is replaced by $z_{j, t-Q_{j}}$. Only constraint (7.10) remains unchanged (compare (6.12)). 
The unique set-up constraint (5.4) and the set-up flow constraint (5.5) are modified to take into consideration the set-up continuation variables:

$$
\begin{aligned}
\sum_{j \in \mathcal{N}}\left(y_{j t}^{\prime}+u_{j t}\right)=1, & t \in \mathcal{T} \\
y_{j t}^{\prime}-y_{j, t-1}^{\prime}+u_{j t}-u_{j, t-1} \leqslant z_{j t}, & t \in \mathcal{T}, j \in \mathcal{N} \\
y_{j, t-1}^{\prime}-y_{j t}^{\prime} \leqslant w_{j t}, & t \in \mathcal{T}, j \in \mathcal{N} \\
y_{j, t-1}^{\prime} \geqslant w_{j t}, & t \in \mathcal{T}, j \in \mathcal{N}
\end{aligned}
$$

Constraint (7.11) ensures an unambiguous state of the machine, which may be either busy with a set-up operation, i.e. $u_{j t}=1$, or ready to produce, i.e. $y_{j t}^{\prime}=1$. Constraint (7.12) forces the start-up variable to take the value 1 in a period with a changeover.

The two next constraints retain the correct values in the switch-off variable. First, (7.13) forces $w_{j t}$ to take on the the value 1 if processing of product $j$ is switched off. Next, (7.14) prevents $w_{j t}$, and indirectly $b_{j t}$, from taking non-zero values in periods where production of product $j$ is not switched off. It should be noticeed that (7.14) was only a valid inequality in the PLSP/E model while here it is an essential part of the model.

In the current model the state of the machine is described by two variables $y_{j t}^{\prime}$ and $u_{j t}$. Therefore valid inequality (1.10) now takes different forms for products with $Q_{j} \geqslant 1$ and $Q_{j}=0$ :

$$
\begin{array}{ll}
z_{j t} \leqslant u_{j t}, & t \in \mathcal{T}, j \in \mathcal{N}: Q_{j} \geqslant 1 \\
z_{j t} \leqslant y_{j t}^{\prime}+u_{j t}, & t \in \mathcal{T}, j \in \mathcal{N}: Q_{j}=0
\end{array}
$$

The reason is that for products with $Q_{j}=0$, production may be possible already in the changeover period but never for products with $Q_{j} \geqslant 1$. However, it should be noted that (7.15) is a special case of (7.8) and therefore may be omitted. Valid inequality (1.11) can be applied here unchanged.

Inventory lower bound constraints (6.14) may now be simplified, because production in many periods is here prevented by set-up continuation variables $u_{j t}$ and set-up state variables $y_{j t}^{\prime}$ precisely denote periods when production is allowed. For product with $Q_{j}=0$, the new inequality has following form:

$$
\begin{aligned}
I_{j, t-1} \geqslant \sum_{s=t}^{t+p} d_{j s}\left[1-y_{j, t-1}^{\prime}-\sum_{r=t}^{s} z_{j, r-1}-\left(z_{j s}-u_{j s}\right)\right] \\
\quad t \in \mathcal{T}, p=0, \ldots, T-t, j \in \mathcal{N}: Q_{j}=0
\end{aligned}
$$

In (7.17), expression $z_{j s}-u_{j s}$ replaces $v_{j s}$ from (6.14).

Because of the dual role of the set-up continuation variables, described after constraint (7.1), this expression cannot be used for products with $Q_{j} \geqslant 1$ : 


$$
\begin{aligned}
& I_{j, t-1} \geqslant \sum_{s=t}^{t+p} d_{j s}\left[1-y_{j, t-1}^{\prime}-\sum_{r=t}^{s} z_{j, r-\left(Q_{j}+1\right)}-z_{j, s-Q_{j}}\right] \\
& t \in \mathcal{T}, p=0, \ldots, T-t, j \in \mathcal{N}: Q_{j} \geqslant 1
\end{aligned}
$$

The lack of a set-up finish variable makes (7.18) less precise than (6.14).

\subsection{EXPLICIT PLSP MODEL WITH CONTINUATION AND FINISH VARIABLES}

It is an appealing idea to build yet another model using both, set-up continuation and set-up finish variables, in the hope, that the advantages arising from a precise formulation may dominate over the cost of the increase in model size. Below, such a model is presented and is denoted PLSP/E-MS/CF. To make the description more concise, only differences between the E-MS/C and E-MS/CF models are described in detail.

Constraint (7.1) has to be substituted with the two following constraints:

$$
\begin{array}{ll}
a_{j t}-F_{j} \leqslant v_{j t}, & j \in \mathcal{N}, t \in \mathcal{T}: t \leqslant T-Q_{j} \\
z_{j t}-v_{j t} \leqslant u_{j, t+Q_{j}}, & j \in \mathcal{N}, t \in \mathcal{T}: t \leqslant T-Q_{j}
\end{array}
$$

Inequality (8.1) is identical to (6.3) from the E-MS/F model. Constraint (8.2) determines the value of the set-up continuation variable in period $t+Q_{j}$ if there was a changeover in period $t$. Because of the dual role of the set-up continuation variables, described after constraint (7.1), constraint (8.2) cannot take the form of an equality.

For products with set-up times shorter than the period, i.e. with $Q_{j}=0$, the capacity constraint (6.1) from the E-MS/F model can be used with one change. If the set-up operation overlaps two periods, then in the first period set-up state variables $y_{j t}^{\prime}$ is now equal to zero and does not increase the value of the right-hand side of the constraint. Only if the whole set-up is executed within the changeover period is it necessary to cancel its value. This can be achieved with the set-up finish variable:

$$
x_{j t}+s_{j t} \leqslant y_{j t}^{\prime}-v_{j t}+b_{j t}+a_{j t}, \quad t \in \mathcal{T}, j \in \mathcal{N}: Q_{j}=0
$$

To determine the values of the set-up execution variables, constraints $(6.5)-(6.7)$ can be applied from the E-MS/F model without modification. This is possible because both models use the set-up finish variables.

To limit the values of the production and set-up execution variables for products with set-up times longer than the period, i.e. $Q_{j} \geqslant 1$, the constraints defined for the E-MS/C model can be used, i.e. (7.7)-(7.10). Constraints preserving the flow of the set-up state (7.11)-(7.14) from the E-MS/C model can also remain unchanged. This is possible because both models use set-up continuation variables.

The following valid inequalities in the current model may be applied without modification: (1.11) from the basic PLSP model, (6.13) from the E-MS/F model and (7.16) from the E-MS/C model. 
Inventory lower bound constraints (7.18) from the E-MS/C model can be tightened in the current model for products with $Q_{j} \geqslant 1$. The set-up finish variable $v_{j, s-Q_{j}}$ now replaces $u_{j, s-Q_{j}}$, in an identical manner to the E-MS/F model:

$$
\begin{aligned}
I_{j, t-1} \geqslant \sum_{s=t}^{t+p} d_{j s}\left[1-y_{j, t-1}^{\prime}-\sum_{r=t}^{s} z_{j, r-\left(Q_{j}+1\right)}-v_{j, s-Q_{j}}\right] & \\
& t \in \mathcal{T}, p=0, \ldots, T-t, j \in \mathcal{N}
\end{aligned}
$$

\section{COMPUTATIONAL RESULTS}

To test the computational properties of all models that have been presented, several problem instances have been solved. The first set of 80 instances was prepared by Suerie (2006). All these instances have a number of periods $T=15$ or $T=30$, constant capacity $C=100,3$ products, production coefficients $p_{j}=1$ for each product, vectors of unit holding costs $h=(1,2,3)$ and set-up costs $S C=(400,800,1200)$.

Three groups of Suerie's (2006) instances have the same relative set-up time for all products $S T_{j}=0.4,0.8$ or 1.2. The fourth group contains mixed cases, where the vector of relative set-up times is equal to $S T=(0.4,0.8,1.2)$.

In tight instances, demand for each product is equally distributed between 10 and 30, whereas in loose instances, demand is equally distributed between 8 and 24 . To make the test examples feasible, demand is set to zero in periods $1-3$ for instances with $T=15$ periods, and in periods $1-5$ for instances with $T=30$ periods. Five different demand series are generated for each of the two planning horizons.

The second set of instances, denoted EA, is based on 2 real demand profiles from the electronic and automobile industry with 30 periods. The profile from the electronic industry contains 3 products (Kaczmarczyk et al., 2006), and the profile from the automobile industry contains 2 products (Miodońska, 2006). Set-up parameters are identical for all products $S T_{j}=0.7,1.4$ or $2.1, S C_{j}=100,300$ or 500 . Unit holding cost is equal to $h=100 / C$. In this way, the smallest tested set-up cost was equal to the inventory holding cost of daily production.

The time bucket size (capacity $C$ ) has been derived from the following definition of utilization:

$$
u=\sum_{j \in \mathcal{N}} \sum_{t \in \mathcal{T}} p_{j} d_{j t} /(T C-3 n S T C)
$$

where the utilization is set to $u=50 \%, 60 \%, 70 \%$ or $80 \%$. The total capacity in this formula is decreased by 3 set-up times per product. The assumed number of start-ups was chosen arbitrarily after a few experiments.

Actual average utilization in all EA instances is presented in Table 7. The goal of such an instance generation procedure was to preserve a similar capacity for processing products at the same number of changeovers independent of set-up length. Actual results show that this does not really succeed. 
The actual utilization decreases significantly with each increment of set-up time. The reason is that, for instances with long set-up times, decreasing the number of start-ups is more profitable as it frees more capacity.

Table 7. Actual utilization in EA instances

\begin{tabular}{cccc}
\hline Assumed & \multicolumn{3}{c}{ Set-up times } \\
utilization & 0.7 & 1.4 & 2.1 \\
\hline $50 \%$ & $51 \%$ & $47 \%$ & $39 \%$ \\
$60 \%$ & $61 \%$ & $58 \%$ & $49 \%$ \\
$70 \%$ & $70 \%$ & $66 \%$ & $57 \%$ \\
$80 \%$ & $79 \%$ & $71 \%$ & $65 \%$ \\
\hline
\end{tabular}

All models described in this paper have been used to solve both sets of test instances. In all models, the number of valid inequalities setting inventory lower bounds, similar to (1.12), was constrained by the parameter $p \leqslant 5$. This limit has been set arbitrarily after a limited number of experiments with some EA instances to find a good balance between model tightness and size.

All tests have been performed on an Intel T1300 processor with a $1.66 \mathrm{MHz}$ clock speed and 1 GB RAM running ILOG OPL Studio 6.3 (CPLEX 12) with standard settings. Computation time was limited to 600 seconds.

In Tables 8 and 9 , the results for all models are presented. Here, the MIP integrality gap is defined as $\left(f^{*}-L B\right) / L B$, where $f^{*}$ is the best value found for the objective function, and $L B$ is the lower bound. MIP gap is equal to zero or very small for all models because most instances have been solved to optimality.

Table 8. Average results for Suerie instances

\begin{tabular}{ccrrrrrr}
\hline & $S T$ & POST2 & POST1 & E-MS/F & E-MS/C & E-MS/CF & CSLP \\
\hline Total cost & 0.4 & 0.0 & 0.0 & 0.0 & 0.1 & 0.0 & 11.4 \\
increment $\dagger$ & 0.8 & 0.0 & 0.1 & -0.1 & 0.5 & 0.1 & 5.5 \\
[\%] & 1.2 & 0.0 & -0.3 & -0.3 & -0.3 & -0.3 & 19.5 \\
& mixed & 0.0 & 0.6 & -0.1 & 0.7 & 0.5 & 12.2 \\
MIP Gap $\ddagger$ & 0.4 & 0.0 & 0.6 & 0.0 & 1.4 & 0.2 & 0.0 \\
[\%] & 0.8 & 2.0 & 5.3 & 0.6 & 4.8 & 2.7 & 0.0 \\
& 1.2 & 6.4 & 0.6 & 0.3 & 0.0 & 0.0 & 0.0 \\
& mixed & 2.9 & 2.1 & 0.6 & 1.5 & 0.5 & 0.0 \\
Time & 0.4 & 55 & 110 & 67 & 150 & 115 & 4 \\
[sec] & 0.8 & 153 & 157 & 154 & 175 & 165 & 9 \\
& 1.2 & 153 & 100 & 116 & 28 & 25 & 5 \\
& mixed & 153 & 147 & 118 & 150 & 122 & 7 \\
\hline
\end{tabular}

$\dagger$ Increment of total costs versus results of the POST2 model $\ddagger$ Relative difference between best value found and lower bound 
Table 9. Average results for EA instances

\begin{tabular}{ccrrrrrr}
\hline & $S T$ & POST2 & POST1 & E-MS/F & E-MS/C & E-MS/CF & CSLP \\
\hline Total cost & 0.7 & 0.0 & 0.4 & 0.0 & 0.3 & 0.1 & 9.5 \\
increment $\dagger$ & 1.4 & 0.0 & 0.0 & -0.1 & 0.0 & 0.0 & 12.5 \\
[\%] & 2.1 & 0.0 & 0.0 & 0.0 & 0.0 & 0.0 & 20.3 \\
MIP Gap $\ddagger$ & 0.7 & 0.1 & 6.0 & 0.3 & 3.7 & 1.4 & 0.0 \\
[\%] & 1.4 & 1.7 & 0.6 & 0.0 & 0.0 & 0.0 & 0.0 \\
& 2.1 & 0.6 & 0.0 & 0.0 & 0.0 & 0.0 & 0.0 \\
Time & 0.7 & 95 & 318 & 110 & 290 & 186 & 2.0 \\
[sec] & 1.4 & 281 & 110 & 59 & 16 & 13 & 0.6 \\
& 2.1 & 147 & 16 & 11 & 4 & 4 & 0.4 \\
\hline
\end{tabular}

$\dagger$ Increment of total costs versus results of the POST2 model $\ddagger$ Relative difference between best value found and lower bound

The higher MIP gap and longer computation times for instances with set-up times shorter than the period show that such instances are harder to solve. For such problems, the most appropriate seems to be the POST2 model which always achieves the best results. However the E-MS/F model is also quite good.

For instances with set-up times longer than the period, the new models, i.e. E$\mathrm{MS} / \mathrm{C}$ and E-MS/CF, are clearly the easiest to solve. Their advantages become more visible when set-up times become longer.

The objective function values are different in the various models for two reasons. Firstly, not all instances are solved to optimality. Secondly, some of the models are more restrictive than others. Some of these differences are described in the previous sections. It seems that these differences do not have a significant impact on the value of the objective function.

The PLSP model is more flexible than the CSLP model because it allows production before the set-up operation. Total cost of the CSLP-LST might even be $20 \%$ higher than for any PLSP model which fully justifies the extra effort to apply the PLSP instead of the CSLP model.

For problems with set-up times shorter than a period, i.e. $Q_{j}=0$, executing of a set-up operation during two periods is another kind of flexibility (Suerie, 2005). To evaluate the impact of these two kinds of flexibility, instances with $Q_{j}=0$ have been solved with the standard PLSP model (1), which assumes that every set-up operation is executed within a single period. These results have been compared with results from the PLSP-POST2 (3) and CSLP-LST (4) models.

On average, the relative additional cost of the standard PLSP and CSLP-LST models compared to the PLSP-POST2 model is presented in Table 10. Both kinds of flexibility seem to have significant impact on total cost. This impact becomes stronger with decreases in set-up cost, because changeovers become more frequent and this creates more opportunity to utilize additional flexibility. 
Table 10. Additional cost of simple models for $S T=0.7$

\begin{tabular}{crr}
\hline$S C$ & CSLP-LST & PLSP \\
\hline 100 & $14.9 \%$ & $8.4 \%$ \\
300 & $7.4 \%$ & $4.1 \%$ \\
500 & $6.2 \%$ & $3.6 \%$ \\
\hline Average & $9.5 \%$ & $5.3 \%$ \\
\hline
\end{tabular}

\section{CONCLUSION}

In this paper, two new mixed integer programming models are presented of the Proportional Lot-Sizing Problem (PLSP) with set-up operations overlapping multiple periods. The basis for the new formulations constitutes a PLSP formulation which explicitly determines the division of time before and after every product changeover. Thanks to the variables describing these times, models that have been presented directly determine the distribution of set-up times over periods.

Computational results show that the proposed models can be solved with standard MIP methods faster than models already known, especially for set-up times longer than the period.

\section{REFERENCES}

Belvaux, G., Wolsey, L.A. (2001). Modelling practical lot-sizing problems as mixed-integer programs, Management Science 47(7): 993-1007.

Blocher, J.D., Chand, S., Sengupta, K. (1999). The changeover scheduling problem with time and cost considerations: Analytical results and a forward algorithm., Operations Research 47: 559-569.

Cattrysse, D., Salomon, M., Kuik, R., van Wassenhove, L. (1993). A dual ascent and column generation heuristic for the discrete lotsizing and scheduling problem with setup times, Management Science 39: 477-486.

Drexl, A., Haase, K. (1995). Proportional lotsizing and scheduling, International Journal of Production Economics 40: 73-87.

Drexl, A., Kimms, A. (1997). Lot sizing and scheduling - survey and extensions, European Journal of Operational Research 99(2): 221-235.

Haase, K. (1994). Lotsizing and scheduling for production planning, number 408 in Lecture Notes in Economics and Mathematical Systems, Springer-Verlag.

Jans, R., Degraeve, Z. (2008). Modeling industrial lot sizing problems: a review, International Journal of Production Research 46(6): 1619-1643.

Kaczmarczyk, W. (2009). Planowanie wielkości i szeregowanie partii z długimi czasami przezbrojeń (Lot-sizing and scheduling with long set-up times), Automatyka 13(2): 315323. in Polish.

Kaczmarczyk, W. (2010). Proportional lot-sizing and scheduling problem with identical parallel machines, International Journal of Production Research accpeted for publication.

Kaczmarczyk, W., Sawik T., Schaller, A., Tirpak, T. (2006). Production planning and coordination in customer driven supply chains, Wybrane Zagadnienia Logistyki Stosowanej, number 3, Komitet Transportu Polskiej Akademii Nauk, pp. 81-89. 
Kimms, A., Drexl, A. (1998). Proportional lot sizing and scheduling: Some extensions, Networks 32(2): 85-101.

Miodońska, B. (2006). Koordynacja w tańcuchach dostaw (Coordination in supply chains), Master's thesis, AGH University of Science and Technology, Krakow, Poland, in Polish.

Pochet, Y., Wolsey, L.A. (2006). Production planning by mixed integer programming, Series in Operations Research and Financial Engineering, Springer, New York.

Suerie, C. (2005). Time Continuity in Discrete Time Models, Springer.

Suerie, C. (2006). Modeling of period overlapping setup times, European Journal of Operational Research 174: 874-886.

Williams, H.P. (2006). Model building in Mathematical Programming, Wiley.

Wolsey, L.A. (2002). Solving multi-item lot-sizing problems with an mip solver using classification and reformulation, Management Science 48(12): 1587-1602. 